\title{
Regional Wall Motion Abnormalities and Ventricular Function in Acute Peri-Myocarditis
}

\author{
Claudia Matei ${ }^{1}$, András Mester ${ }^{2}$, Daniel Cernica², Roxana Hodas ${ }^{2}$, Lehel Bordi² \\ 1 "Dr. C-tin Opriș" County Emergency Clinical Hospital, Baia Mare, Romania \\ 2 County Emergency Clinical Hospital, Tîrgu Mureș, Romania
}

\section{ABSTRACT}

Introduction: Myocardial involvement in pericardial diseases and the effect of pericardial inflammation and effusion on the function of the left ventricle (LV) is still a controversial issue. The present study aimed to evaluate LV regional wall abnormalities in patients with acute peri-myocarditis, using 3D echocardiographic assessment of LV contractility. Material and methods: The study included 56 subjects divided into two groups: Group $1-28$ subjects with acute peri-myocarditis and Group $2-28$ healthy controls. All subjects underwent a complete clinical examination, including laboratory tests. 3D echocardiography was performed to assess the left ventricular end-diastolic (EDV) and end-systolic volumes (ESV), and to calculate specific indexes for global and regional ventricular contractility, such as the index of contraction amplitude (ICA) and the regional index of contraction amplitude (RICA) for each segment corresponding to the region affected by peri- myocarditis. Results: 3D echocardiography showed no differences between the groups regarding the EDV $(\mathrm{p}=0.2)$, the LV ejection fraction (Group 1: $55.82 \% \pm 3.36 \%$ vs. Group $2: 57.21 \% \pm 4.69 \%, p=0.2)$. The ESV however, was significantly higher in Group 1 compared to Group $2(55.78 \pm 5.45 \mathrm{ml}$ vs. $52.20 \pm 6.43 \mathrm{ml}, \mathrm{p}=0.04)$. ICA was similar between the groups ( $\mathrm{p}=0.2$ ). However, the RICA was significantly lower in Group 1 compared to Group 2 ( $2.27 \pm 0.63$ vs. $5.16 \pm 0.54, p<0.0001)$. Conclusions: The extension of the inflammatory process from the pericardium to the adjacent myocardial layer may affect the contractility of the LV. A good association exists between peri-myocarditis and the regional contractility abnormalities of the LV.

Keywords: peri-myocarditis, echocardiography, original index of contraction, amplitude

\section{ARTICLE HISTORY}

Received: 14 February, 2017

Accepted: 16 March, 2017
Claudia Matei

Str. Gheorghe Coșbuc nr. 31 430031 Baia Mare, Romania Tel: +40 740085662

E-mail: cabdia@yahoo.com

\section{INTRODUCTION}

Pericardial diseases represent one of the most underdiagnosed cardiovascular conditions in clinical practice due to the absence of well-established diagnostic criteria. ${ }^{1,2}$ This condition has been reported in approximately $1 \%$ of hospitalized patients and $5 \%$ of necropsies per- formed in general hospitals. The most common causes of acute pericarditis are represented by a viral infection, frequently labeled as idiopathic due to the difficult identification of the involved virus, followed by bacterial or parasitic infections. ${ }^{3}$ Systemic inflammatory diseases, neoplasms, and traumas may represent the underlying cause of the acute inflammation of the peri- and myo- 
cardium. ${ }^{4}$ Although pericarditis is considered a benign condition, its acute complications, such as cardiac tamponade or involvement of the myocardial layer, may lead to life-threatening conditions such as acute heart failure or death. ${ }^{5}$ However, although a vast number of studies have investigated the clinical aspects of pericardial diseases, surprisingly few of them have analyzed the extension of the inflammatory process from the pericardium into the myocardium. ${ }^{6}$

The first-line diagnostic means for the recognition of peri-myocardial diseases are echocardiography and cardiac magnetic resonance (CMR), whereas computed tomography (CT) is considered a second-line option. ${ }^{7,8}$ The recent improvement in different diagnostic techniques, notably echocardiography, and longer survival times have led to an increase in the recognition of pericardial diseases in recent years. ${ }^{9}$

The current literature mentions the possible effects of pericardial effusion accumulation on the general function of the heart, however, it does not state its exact influence on the regional wall motion abnormalities of the left ventricle (LV).

Our hypothesis is that the extension of the inflammatory process from the pericardium to the myocardial layer in acute peri-myocarditis may lead to the alteration of ventricular wall motion, involving the regions near to the affected pericardium.

The aim of our study was to evaluate regional ventricular wall motion abnormalities in patients with acute perimyocarditis using three-dimensional echocardiography.

\section{MATERIAL AND METHODS}

\section{STUDY DESIGN}

This is a prospective observational study, conducted at the Cardiology Clinic of the County Emergency Clinical Hospital of Tîrgu Mureș, Romania, in which fifty-six subjects were enrolled and divided into two groups: Group $1-28$ patients diagnosed with acute peri-myocarditis; Group 2 - 28 healthy subjects, without any signs or symptoms of peri-myocarditis. The study protocol was approved by the Ethical Committee of the County Emergency Clinical Hospital of Tîrgu Mureș, Romania, and all patients signed a written informed consent form before enrollment.

\section{INCLUSION CRITERIA}

The inclusion criteria were the presence of clinical symptoms of acute chest pain that worsens on breathing or when changing the position of the body, associated with at least one of the following:

- pericardial friction rub;

- PR- or ST-segment modifications on the 12-lead electrocardiogram (ECG), suggestive for pericarditis;

- evidence of pericardial effusion/thickening of the pericardium with at least $5 \mathrm{~m}$ on transthoracic ECG.

\section{EXCLUSION CRITERIA}

The exclusion criteria were:

- patients presenting with atherosclerotic or thrombotic coronary lesions;

- unstable angina;

- post-infarction angina;

- recent myocardial infarction;

- congestive heart failure;

- cancers;

- history of heart surgery;

- NYHA functional class 4;

- severe valve disease;

- severe renal, hepatic, hematologic or pulmonary disease;

- age below 18 years.

\section{METHODOLOGY}

\section{Clinical data}

The history and clinical data of all enrolled subjects were recorded, including age, gender, cardiovascular risk factors (diabetes mellitus, hypertension, dyslipidemia, smoking, family history of cardiovascular diseases), and personal history (stable/unstable angina, myocardial infarction, ischemic heart disease, stroke).

\section{Paraclinical data}

Standard 12-lead resting ECG was obtained from all patients, documenting the existence of any abnormality of the repolarization phase.

Echocardiography was performed using an Agilent Sonos 4500 ultrasound imaging system (Philips, Amsterdam, the Netherlands), and the following parameters were recorded:

- pericardial thickening;

- amount and location of pericardial fluid; 
- left ventricular volumes;

- left ventricular ejection fraction;

- contraction abnormalities.

Three-dimensional echocardiography was also performed for the assessment of the following:

- total and regional left ventricular ejection fraction;

- detection of regional wall motion abnormalities;

- calculation of the end-diastolic and end-systolic volume;

- evaluation of the regions with wall motion abnormalities (contraction asynchronies);

- measurement of the delay in maximal global systolic contraction amplitude, assessment of the index of contraction amplitude (ICA);

- regional index of contraction amplitude (RICA);

- the volumetric curve for each ventricular region;

- polar mapping of the delay in maximal systolic contraction amplitude.

For patients in Group 2, the contractility index was calculated for each ventricular region corresponding to the region affected by peri-myocarditis compared to the regions without peri-myocarditis.

As the clinical, laboratory, and ECG findings in acute peri-myocarditis may be similar to ischemic coronary disease, invasive coronary angiography was performed in cases when an acute coronary syndrome could not be ruled out by noninvasive examination.

The study has been carried out in accordance with the code of ethics of the World Medical Association's Declaration of Helsinki. All patients gave written informed consent, and the study protocol was approved by the ethics committee of the institution where the study was conducted.

\section{STATISTICAL ANALYSIS}

JMP statistical software was used for statistical analysis. The Mann-Whitney test was used for continuous values expressed as means (SD). Fisher's exact test or the Chisquare test was used for the comparison of categorical variables expressed as percentages. The level of significance was set at $\alpha=0.05$.

\section{RESULTS}

\section{BASELINE AND CLINICAL CHARACTERISTICS}

There was no significant difference between the two study groups regarding age, with a mean of $55.28 \pm 11.28$ years in Group 1 vs. $53.6 \pm 10.51$ years in Group $2(\mathrm{p}=$ $0.5)$. There was a preponderance of female subjects in both study groups $(\mathrm{p}=0.1)$. The presence of cardiovascular risk factors and comorbidities was similar in the two study groups in respect of diabetes mellitus ( $p=0.7)$, hypertension $(\mathrm{p}=0.5)$, obesity $(\mathrm{p}=0.7)$, and smoking ( $\mathrm{p}$ $=0.2)$ (Table 1).

\section{ECHOCARDIOGRAPHIC FINDINGS}

M-mode and two-dimensional echocardiography revealed thickening of the pericardium with a mean of $55.28 \pm 11.28$ years in Group 1 vs. $53.6 \pm 10.51$ years in Group $2(p=0.5)$ in patients from Group 1. The localization of the pericardial effusion was mainly in the lateral region of the left ventricle $(n=12,42.8 \%)$, followed by the inferior region of the left ventricle $(n=10,35.7 \%)$, and the apical region ( $\mathrm{n}=$ 6, 21.4\%). M-mode and two-dimensional echocardiography showed no pathological findings in Group 2.

Three-dimensional echocardiographic analysis of the global and regional contractility showed no significant difference between the two study groups in terms of end-diastolic volume (EDV), with a mean of $123.14 \pm$

TABLE 1. Baseline characteristics of the study groups

\begin{tabular}{lccc}
\hline Laboratory findings & $\begin{array}{c}\text { Group 1 } \\
\mathbf{n = 2 8}\end{array}$ & $\begin{array}{c}\text { Group 2 } \\
\mathbf{n = 2 8}\end{array}$ & p value \\
\hline Age & $55.28 \pm 11.28$ & $53.60 \pm 10.51$ & 0.5 \\
Female gender, n (\%) & $19(67.85 \%)$ & $18(64.28 \%)$ & 0.1 \\
Diabetes mellitus, n (\%) & $9(32.14 \%)$ & $7(25.00 \%)$ & 0.7 \\
Hypertension, n (\%) & $10(35.71 \%)$ & $7(25.00 \%)$ & 0.5 \\
Obesity, n (\%) & $5(17.85 \%)$ & $7(25.00 \%)$ & 0.7 \\
Smoking (past or present), n (\%) & $11(39.28 \%)$ & $8(28.57 \%)$ & 0.2 \\
\hline
\end{tabular}




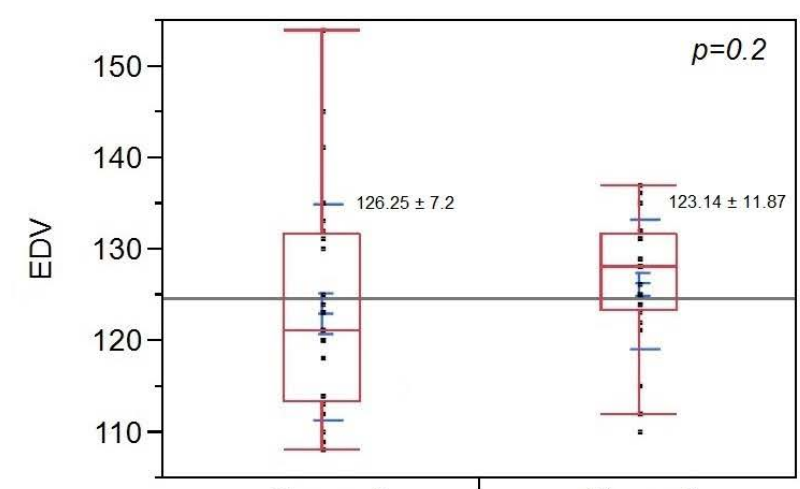

A

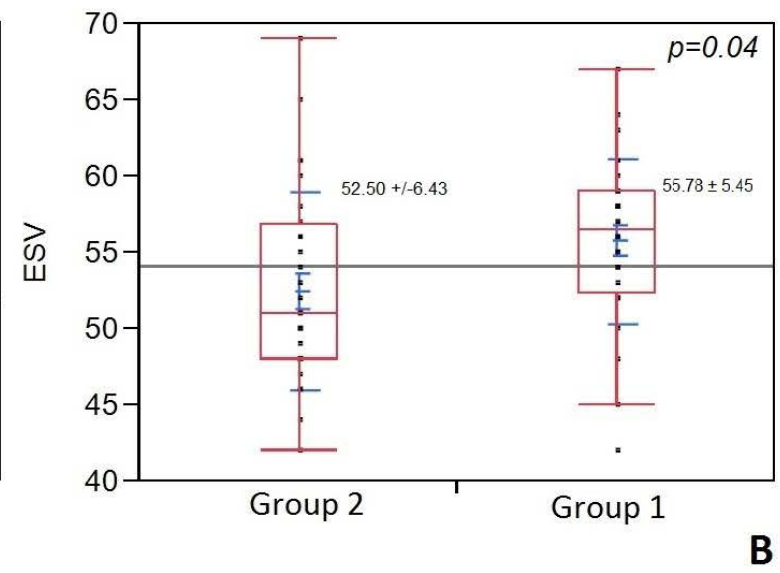

FIGURE 1. A- End-diastolic volume of the study groups. B - End-systolic volume of the study groups

$11.87 \mathrm{ml}$ in Group 1 vs. $126.25 \pm 7.2 \mathrm{ml}$ in Group $2(\mathrm{p}=0.2)$ (Figure $1 \mathrm{~A}$ ).

The end-systolic volume (ESV) was significantly higher in patients from Group 1 with $55.78 \pm 5.45 \mathrm{ml}(95 \% \mathrm{CI}$ : 53.67-57.90) vs. $52.50 \pm 6.43 \mathrm{ml}(95 \% \mathrm{CI}: 50.00-54.99)$ in Group 2 ( $\mathrm{p}=0.04)$ (Figure $1 \mathrm{~B})$.

There was no significant difference in respect of the calculated global ejection fraction (EF) between the study groups, with $55.82 \pm 3.36 \%$ (95\% CI: 54.52-57.13) in Group 1 vs. $57.21 \pm 4.69 \%$ (95\% CI: 55.39-59.03) in Group $2(\mathrm{p}=0.2)$ (Figure 2$)$.

ICA was similar in the two study groups with a value of $3.22 \pm 0.68$ (95\% CI: 2.92-3.53) for patients with acute peri-myocarditis vs. $3.93 \pm 0.33$ (95\% CI: $3.76-4.11$ ) in the control group ( $\mathrm{p}=0.2)$.

RICA was significantly lower in the proximity of the affected peri-myocardium in patients with acute peri-myocarditis in Group 1, with a mean of $2.27 \pm 0.63$ (95\% CI: 2.02-2.51) vs. $5.16 \pm 0.54$ (95\% CI: 4.95-5.37) in Group 2 $(\mathrm{p}<0.0001)$ (Figure 3$)$.

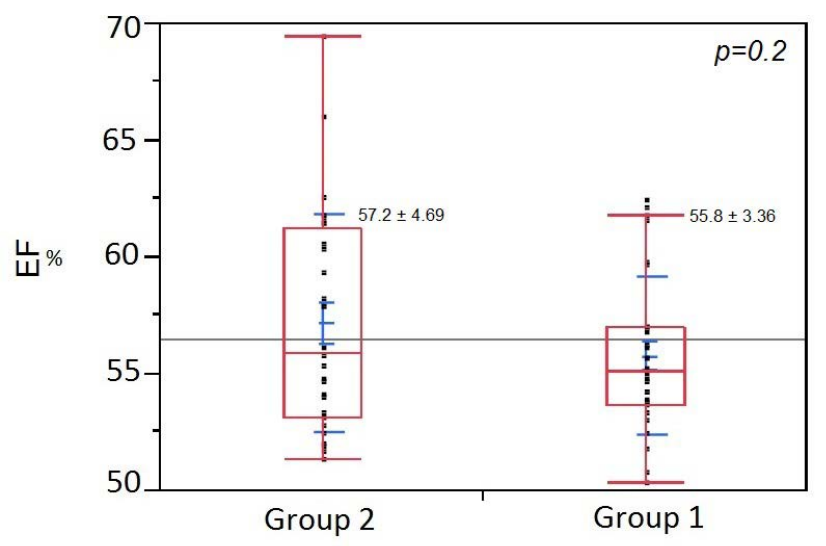

FIGURE 2. Ejection fraction of the study groups

\section{DISCUSSION}

Peri-myocarditis can occur at any age, with peak prevalence middle-aged subjects. The mean age of our study population with acute peri-myocarditis was $55.28 \pm 11.28$ years. Some authors suggest that there may be a difference in respect of age in patients presenting with acute pericarditis versus those showing associated myocardial affection. $^{10}$

The literature data is conflicting regarding the sex-related risk of developing peri-myocarditis. ${ }^{11,12}$ In an experimental study, Frisancho-Kiss et al. (2009) reported on the role of testosterone in the development of myocarditis. ${ }^{13}$ In the present study female gender was predominant in patients with acute peri-myocarditis (67.85\%). These results may be explained by the fact that systemic inflammatory diseases are more common in females, and some authors suggest that progesterone may aggravate myocardial inflammation, though estrogen inhibits the immune response. ${ }^{14,15}$

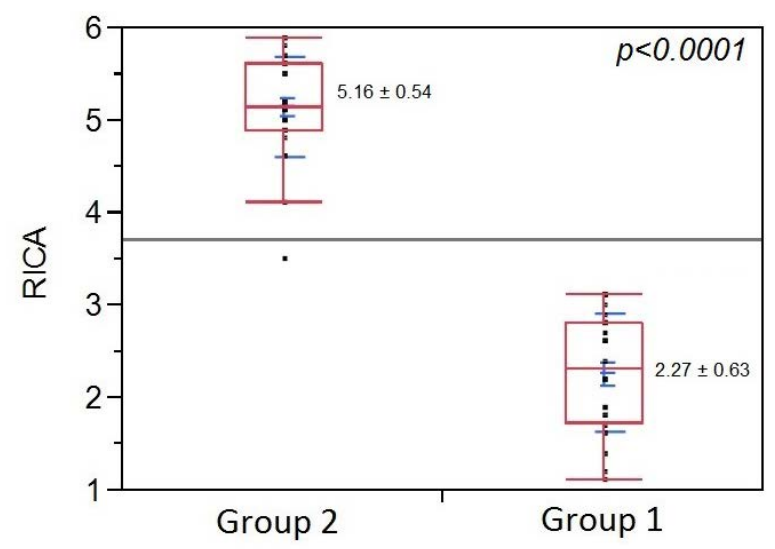

FIGURE 3. Regional index of contraction amplitude (RICA) 
No significant differences were recorded between the two groups in terms of associated comorbidities such as diabetes mellitus $(p=0.7)$, hypertension $(p=0.5)$, obesity ( $\mathrm{p}=0.7)$, and smoking $(\mathrm{p}=0.2)$, suggesting that these well-known cardiovascular risk factors do not have an influence on the inflammatory process that is taking place during the acute phase of peri-myocardial disease. ${ }^{16}$

Echocardiography is the first-line diagnostic choice for the identification of acute pericarditis, being a noninvasive, commonly available examination. It has a high accuracy in assessing the hemodynamic and physiologic influences on heart function. ${ }^{8}$

Acute heart failure can be caused by the existence of large myocardial inflammation zones resulting in a reduction of left ventricular ejection fraction, characterizing a rare, but severe complication of peri-myocarditis. ${ }^{17}$ A significant amount of rapidly accumulated pericardial effusion causing cardiac tamponade represents a lifethreatening condition in acute pericarditis, which requires immediate treatment. ${ }^{18}$ In the current study, the thickening of the pericardium and the presence of pericardial effusion, identified by echocardiography, did not have a significant bearing on the performance of the left ventricle expressed by the left ventricular ejection fraction, enddiastolic volume, and end-systolic volume in patients with acute peri-myocarditis. The global ICA, determined by three-dimensional echocardiography, was lower for Group 1 patients with acute peri-myocarditis compared with the control group, although the difference was not significant $(\mathrm{p}=0.2)$. These results are concordant with literature data, as acute peri-myocarditis rarely has a major effect on the overall function of the heart. ${ }^{19}$ In an MRIcontrolled study, Goitein et al. (2009) reported 23 patients presenting acute myocarditis with a mean left ventricular EF of $57 \%$, without any significant decrease in the global function of the left ventricle. ${ }^{20}$

Pericardial effusion and regional wall motion abnormalities were mainly present on the lateral wall of the left ventricle $(42.8 \%)$, followed by the inferior wall $(35.7 \%)$ and the apex (21.4\%). In a study of 100 patients with acute peri-myocarditis, Leitman et al. (2012) reported a predominance of regional wall abnormalities of the posteroinferolateral wall of the LV in the absence of any effect on LV performance. ${ }^{21}$

In the present study, the RICA was significantly lower in the proximity of the affected myocardial areas in Group 1 patients compared with Group 2 ( $p<0.0001)$. This may be explained by the presence of pericardial effusion and the thickening of the pericardium, which has a bearing on the regional wall motion through the extension of the in- flammatory process from the pericardium to the adjacent myocardium but does not affect the overall function of the left ventricle. Speckle-tracking imaging may also identify regional wall motion abnormalities in patients with acute peri-myocarditis. ${ }^{22}$

The hemodynamic impact of pericardial effusions varies from mild to life-threatening, depending on the rate of accumulation and the thickness of the pericardium. Adhesions of the pericardial layers and localized liquid buildup may develop an atypical tamponade.10 However, no patient with cardiac tamponade was recorded in the current study.

\section{CONCLUSIONS}

Detailed echocardiographic evaluation of patients presenting with a possible acute peri-myocarditis should be considered the basis of clinical differentiation of peri-myocardial syndromes. The extension of the inflammatory process from the pericardium to the myocardium in patients with acute peri-myocarditis may have an influence on the contractility of the left ventricle, evaluated by three-dimensional echocardiography based on objective assessment and quantification of contractility parameters. Statistical analysis of an easily measurable objective echocardiographic parameter such as the regional index of contractility amplitude, demonstrated the existence of an association between the presence of peri-myocarditis and the regional contractility abnormalities of the left ventricle.

\section{CONFLICT OF INTEREST}

Nothing to declare.

\section{ACKNOWLEDGEMENT}

This research was supported via the research grant no. 103545/2016, contract number 72/2006, entitled "Translation of genomic research in tridimensional reconstruction and intracardiac imaging in diagnosis and treatment of several congenital or acquired heart diseases in young adult- TRIDICO" financed by Academy of Medical Sciences, Ministry of Education and Research through CEEX 2006 program.

\section{REFERENCES}

1. Imazio M, Gaita F, LeWinter M. Evaluation and Treatment of Pericarditis. A Systematic Review. JAMA. 2015;314:1498-1506. doi: 10.1001/jama.2015.12763. 
2. Lazaros G, Imazio M, Brucato A, Tousoulis D. Untying the Gordian knot of pericardial diseases: A pragmatic approach. Hellenic J Cardiol. 2016;pii:S1109-9666(16)30305-0. doi: 10.1016/j.hjc.2016.11.024.

3. Imazio M, Cecchi E, Demichelis B, et al. Myopericarditis versus viral or idiopathic acute pericarditis. Heart. 2008;94:498-501. doi: 10.1136/hrt.2006.104067.

4. Cantarini L, Imazio M, Brizi MG, et al. Role of autoimmunity and autoinflammation in the pathogenesis of idiopathic recurrent pericarditis. Clin Rev Allergy Immunol. 2013;44:613. doi: $10.1007 / \mathrm{s} 12016-010-8219-\mathrm{x}$.

5. Buiatti A, Merlo M, Pinamonti B, De Biasio M, Bussani R, Sinagra G. Clinical presentation and long-term follow-up of perimyocarditis. J Cardiovasc Med (Hagerstown). 2013;14:235241. doi: 10.2459/JCM.obo13e328351da6e.

6. Chang SA, Kim HK, Kim YJ, Cho GY, Oh S, Sohn DW. Role of pericardium in the maintenance of left ventricular twist. Heart. 2010;96:785-790. doi: 10.1136/hrt.2009.182345.

7. Otto C. Pericardial Disease. In: Textbook of Clinical Echocardiography. 5th edition. Philadelphia: Elsevier Saunders; 2013. p. 254-271.

8. Klein AL, Abbara S, Agler DA, et al. American Society of Echocardiography clinical recommendations for multimodality cardiovascular imaging of patients with pericardial disease. J Am Soc Echocardiogr. 2013;26:965-1012.e15. doi: 10.1016/j. echo.2013.06.023.

9. Jenkins C, Marwick TH. Baseline and follow-up assessment of regional left ventricular volume using 3-dimensional echocardiography: comparison with cardiac magnetic resonance. Cardiovasc Ultrasound. 2009;7:55. doi: 10.1186/1476-7120-7-55.

10. Ville K, Jussi S, Päivi R. Clinical Profile and Influences on Outcomes in Patients Hospitalized for Acute Pericarditis. Circulation. 2014;130:1601-1606. doi: 10.1161/ CIRCULATIONAHA.114.010376.

11. Zayas R, Anguita M, Torres F, et al. Incidence of specific etiology and role of methods for specific etiologic diagnosis of primary acute pericarditis. Am J Cardiol. 1995;75:378-382.

12. Imazio $M$, Bobbio $M$, Cecchi $E$, et al. Colchicine in addition to conventional therapy for acute pericarditis: results of the COlchicine for acute PEricarditis (COPE) trial. Circulation. 2005;112:2012-2016. doi: 10.1161/ CIRCULATIONAHA.105.542738.
13. Frisancho-Kiss S, Coronado MJ, Frisancho JA, et al. Gonadectomy of male BALB/c mice increases Tim-3(+) alternatively activated M2 macrophages, Tim-3(+) T cells, Th2 cells and Treg in the heart during acute Coxsackievirusinduced myocarditis. Brain Behav Immun. 2009;23:649-657. doi: 10.1016/j.bbi.2008.12.002.

14. Lyden DC, Huber SA. Aggravation of coxsackievirus, group B, type 3-induced myocarditis and increase in cellular immunity to myocyte antigens in pregnant Balb/c mice and animals treated with progesterone. Cell Immunol. 1984;87:462-472.

15. Huber SA. Coxsackievirus B3-induced myocarditis: infection of females during the estrus phase of the ovarian cycle leads to activation of T regulatory cells. Virology. 2008;378:292298. doi: 10.1016/j.virol.2008.05.015.

16. Kato S, Morimoto S, Hiramitsu S, et al. Risk factors for patients developing a fulminant course with acute myocarditis. Circ J. 2004;68:734-739.

17. Basman C, Agrawal PR, McRee C, et al. Diagnostic Approach to Myocarditis Mimicking Myocardial Infarction at Initial Presentation. Cardiol Res. 2016;7:209-213. doi: 10.14740/ cr485w.

18. Streuber S, Noack F, Stoevesandt D, Schlitt A. 102 patients with suspected myocarditis: clinical presentation, diagnostics, therapy and prognosis. Herz. 2017. doi: 10.1007/s00059-0164524-3. [Epub ahead of print]

19. Sturmberger T, Niel J, Aichinger J, Ebner C. Acute myocarditis with normal wall motion detected with 2D speckle-tracking echocardiography Echo Res Pract. 2016;3:K15-K19. doi: 10.1530/ERP-16-0013.

20. Goitein O, Matetzky S, Beinart R, et al. Acute myocarditis: noninvasive evaluation with cardiac MRI and transthoracic echocardiography. AJR Am J Roentgenol. 2009;192:254-258. doi: 10.2214/AJR.08.1281.

21. Leitman M, Tyomkin V, Peleg E. Left ventricular function in acute inflammatory peri-myocardial diseases - new insights and long-term follow-up. Cardiovasc Ultrasound. 2012;10:42. doi: 10.1186/1476-7120-10-42.

22. Leitman M, Bachner-Hinenzon N, Adam D, et al. Speckletracking imaging in acute inflammatory pericardial diseases. Echocardiography. 2011;28:548-555. doi: 10.1111/j.15408175.2010.01371.x. 